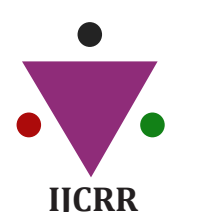

Section: Healthcare

Sci. Journal Impact

Factor: $6.1(2018)$

ICV: 90.90 (2018)

(c) (i) (3)

Copyright@IJCRR

\title{
Blood Donor Notification and Counselling: Experience from a Teaching Hospital in Eastern India
}

\author{
Santosh Mishra ${ }^{1}$, Girijanandini Kanungo ${ }^{2}$, Priyanka Nagrath ${ }^{3}$, Milind Agrawal ${ }^{4}$

\begin{abstract}
1,3.4PG tutor, Transfusion Medicine Department, IMS and SUM Hospital, Sikhsha O Anusandhan, (deemed to be) University, Bhubaneswar, India; 'Associate Professor, Transfusion Medicine Department, IMS and SUM Hospital, Sikhsha O Anusandhan, (deemed to be) university Bhubaneswar, India.
\end{abstract}

\section{ABSTRACT}

Aims: To evaluate the response rate of transfusion transmissible infections (TTI) reactive donors after notifying their abnormal test results at a teaching hospital in eastern India from March 2019 to January 2020.

Materials and Methods: This study is a retrospective analysis performed at our department for a period of 11 months. We evaluated the response rate of the sero-reactive donors towards notification of their abnormal test results as a part of post-donation counselling as per the existing strategy of our institute (one telephonic call followed by two reminder calls within one week) in the mobile number provided by the donor.

Results: During the period of study among 9922 blood donors 201(2.02\%) were sero reactive. Among these the sero-reactive pattern of HIV, HBV, HCV, Syphilis, Malaria were $0.06 \%, 0.65 \%, 0.60 \%, 0.69 \%$, and $0.01 \%$, respectively. After notifying them telephonically about $63.1 \%(n=127)$ could be contacted, advised, and termed Responders. About $36.8 \%$ ( $n=74)$ could not be contacted and termed Non-Responders due to various reasons analyzed. $25.2 \%(n=32)$ reported at our institute for face to face counselling.

Conclusion: Donor notification is an effective method of curtailing TTI but undermined by communication failure with donors, resulting in a persistent load of blood transmissible infectious risk. We need a better structure of pre-donation counselling with the latest and more developed means of communication with our valuable blood donors.

Key Words: Sero-reactive donors, Donor Notification, Responders, Transfusion transmissible infections, Donor Counselling

\section{INTRODUCTION}

Blood Transfusion Services have the vital responsibility of providing the finest possible quality of blood to meet the requirements of the patient. As per the Drugs and Cosmetics Act, 1940, regulations 1945, India all the blood donations are to be screened for the five common Transfusion Transmitted Diseases (TTD) namely HIV I \& II, HBV, HCV, Syphilis and Malaria ${ }^{1}$ NACO (National Aids Control Organisation) guides that ELISA (Enzyme-Linked Immunosorbent Assay) testing kits preferably of Third and Fourth generation bearing $100 \%$ sensitivity to be used to screen the donors for the above said TTD . ${ }^{2}$ The practice of thorough screening, testing, notifying, and counselling of blood donors for transfusion transmissible infection (TTI) is a very important and efficient method of curtailing transfusion-transmitted infections, but the communication of positive test results to blood donors is not a universal procedure. ${ }^{3}$ Conveying the donors about their reactivity status in the right manner stays the backbone of donor notification. National Blood Policy was adopted by the Indian Government in 2002 as "an action plan for blood safety" ensuring the supply of quality blood and blood components. According to the National Blood Policy the test results which are turned out to be abnormal for these TTI should be disclosed to the blood donors. ${ }^{4}$ Due to the paucity of the available literature and information on donor notification in India, this study has been done to find out the prospective of donors toward the disclosure of their TTI reactivity status and to find out the percentage of responders in our population.

\section{Corresponding Author:}

Girijanandini Kanungo, Associate Professor, Transfusion Medicine Department, IMS and SUM Hospital, Sikhsha O Anusandhan, (deemed to be) University, Bhubaneswar, India; Email: gnkanungo@soa.ac.in

ISSN: 2231-2196 (Print)

Received: 06.06.2020
ISSN: $0975-5241$ (Online)

Revised: 20.07 .2020
Accepted: 15.08 .2020 


\section{METHODS}

Retrospective analysis of data was performed in the Department of Transfusion Medicine, IMS and SUM Hospital, Bhubaneswar, a teaching hospital in eastern India from March 2019 to January 2020. In this study, we evaluated the response of the blood donors towards donor notification of sero- reactive cases as a part of post-donation counselling. All the donors were selected for donation as per departmental SOPs and selection criteria of donors as per the Drug and Cosmetic Act, 1945. ${ }^{5}$ For all the voluntary and replacement donors pre-donation medical examination was done to find their eligibility by a medical officer. All donors found eligible were asked to fill a registration form stating their personal details. A trained counsellor performed the pre-donation counselling. The aim of counselling was to make the donors aware of Transfusion Transmissible Infections (TTI) and their prevention. Consent was also obtained from each donor regarding notification of his or her TTI status. All the donations were screened for TTI namely human immunodeficiency virus (HIV) by fourth-generation enzyme-linked immunosorbent assay (ELISA), hepatitis B (HBV), Hepatitis (HCV), Malaria, and Syphilis by third-generation (ELISA) (QUALISA). All the reactive donors were contacted as per the existing strategy of our institute (one telephonic call followed by two reminder calls within one week) on the mobile number provided by the donor to inform about the abnormal results and advised to consult the concerned medical practitioner in their locality or visit the nearest ICTC centre or visit our blood bank. All of them who agreed to the advice with their consent to do so were termed as the Responders. However, the donors who could not be contacted after all the calls were termed the Non-Responders. The reasons found for not being able to contact were calls received by relatives, wrong mobile numbers, and numbers out of reach or switched off mobiles during all the calls. Subsequently, a letter requesting to call or report at the blood bank was also sent to the nonresponders. Among the responders who managed to come to the blood bank, were counselled and referred to appropriate centres for confirmation of their reactive status and further management. Hepatitis B, C, and malaria reactive donors were referred to the medicine OPD, syphilis reactive to the Dermatology and venereal diseases OPD and HIV reactive to the integrated counselling and testing centre (ICTC) of the hospital. Statistical analysis of results recorded was done using SPSS (v23.0)

\section{RESULTS}

There were 9922 blood donations consisting of 9843 (99.2\%) male donors and $79(0.79 \%)$ females over the period of study, out of which $201(2.02 \%)$ were reactive. Among these sero-reactive pattern HIV, HBV, HCV, Syphilis, and Malaria were $0.06 \%, 0.65 \%, 0.60 \%, 0.69 \%$, and $0.01 \%$, respectively as shown in Table 1. No case of co-infection was found. No female reactive donors were found during the study period.

Table 1: Seroreactivity rate of human immunodeficiency virus (HIV), hepatitis B (HBV), hepatitis C (HCV), syphilis and Malaria.

\begin{tabular}{lcc} 
TTI Markers & $\begin{array}{c}\text { Sero-reactive donors } \\
\text { HIV }\end{array}$ & $\begin{array}{c}\text { TTI sero-reactive } \\
\text { rate }\end{array}$ \\
HBV & $6 / 9922$ & $0.06 \%$ \\
HCV & $65 / 9922$ & $0.65 \%$ \\
Syphilis & $60 / 9922$ & $0.60 \%$ \\
Malaria & $69 / 9922$ & $0.69 \%$ \\
\hline
\end{tabular}

Out of all the reactive donors, about $63.1 \%(n=127)$ were the Responders as explained in Figure 1. However, among them, only $25.2 \%(n=32)$ turned up for face to face post donation counselling and referral at our blood bank. About 36.8 $\%(n=74)$ were the Non-responders .Among these 19.9\% $(\mathrm{n}=40)$ calls were received by Relatives, $4.9 \%(\mathrm{n}=10)$ wrong mobile numbers, $6.4 \%(\mathrm{n}=13)$ were out of reach and $5.4 \%$ $(n=11)$ were switched off during all the calls as shown in Figure 2.

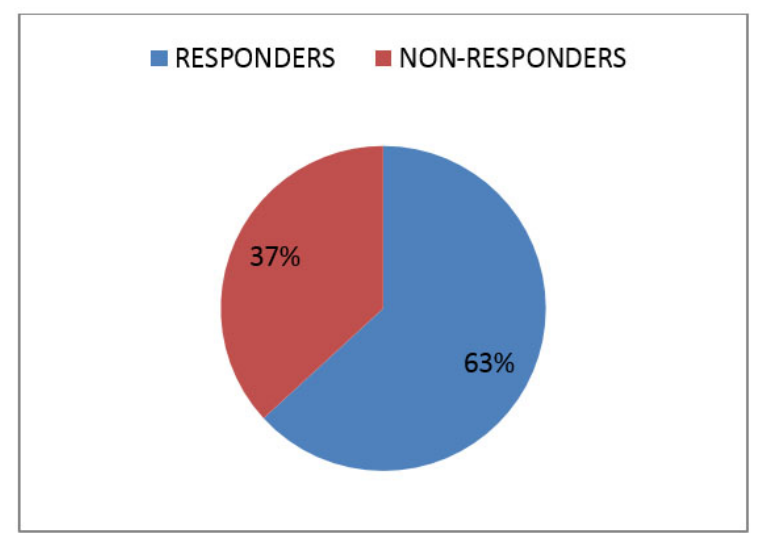

Figure 1: Showing Donor Response to Notification.

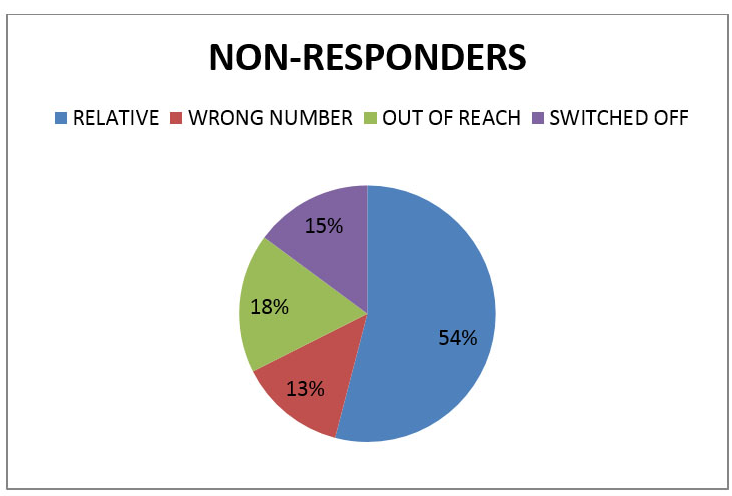

Figure 2: Non-Responders Analysis. 
The response of responders with respect to their age distribution in years is clearly represented in Figure 3.

Most of the responders were found within 18-25 years of age, followed by donors with the age group 26-30 years concluding that majority of the population to be young.

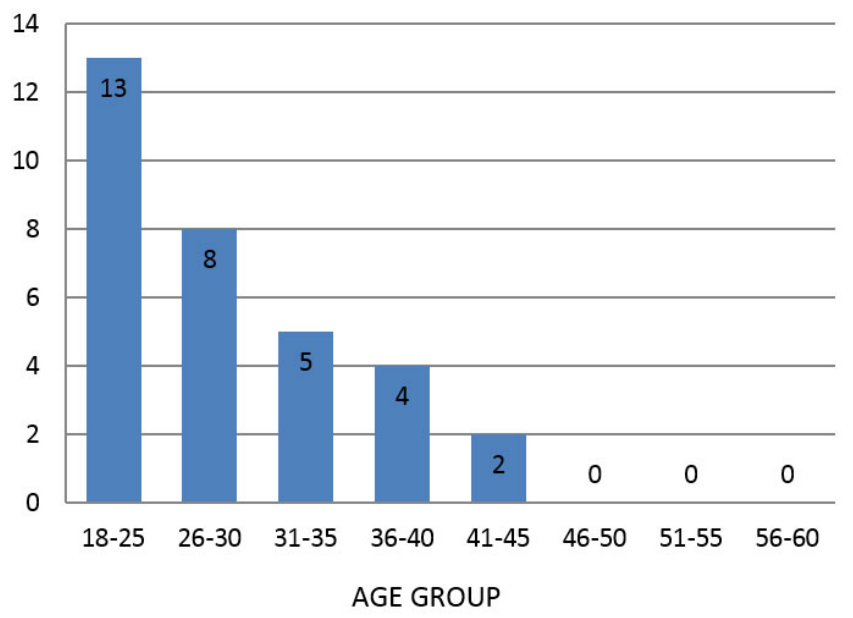

Figure 3: Age-wise distribution of Responders.

\section{DISCUSSION}

A motivated donor donates with a noble intention considering oneself as healthy. Blood donor notification represents a stage in which an individual without any symptom is disclosed about their abnormal test results relating to the exposure of clinically significant infectious agents. Hence, notifying a blood donor about the test results which came out to be abnormal is a crucial and important part of postdonation counselling because of its large psychological and social impact.

Donor notification and post-donation counselling are an essential aspect of the blood bank these days for reactive donors. ${ }^{6}$ The main aim of the notification process is to ensure that donors receive their test results and those found ineligible for future donation are convinced about their deferral status. We found that the notification process does not always achieve these goals as in our data only $63.2 \%$ $[\mathrm{n}=127]$ sero-reactive donors could be contacted successfully. Though $25.2 \%$ [ $n=32$ ] could report at the blood bank for post-donation counselling and referral, a large number about $36.8 \%$ [ $n=74]$ among the reactive donors could not be contacted. In a study conducted in Rockville, MD, USA, an anonymous survey was conducted of blood donors with an abnormal infectious disease screening result. The survey had a $42 \%$ response rate, $10 \%$ of the donors did not recall being notified of their results and only $27 \%$ contacted the blood bank for further information. ${ }^{7}$ The lower response rate of our donors suggests less awareness about the health issues and a low concept about the screening of donors. A similar response rate $(21-67 \%)$ is also seen in a study of Moyer et al. ${ }^{8}$ Similarly the study conducted by Kaur et al. ${ }^{9}$ showed that the decreased response in their donors might attribute to low health care knowledge and poor understanding about screening results. A study by Agarwal ${ }^{10}$ showed that $59.8 \%$ were responders. A study in Kerala reports that $53 \%$ of the donors were well-versed about the donor notification process however, $88.3 \%$ could never be contacted. ${ }^{11}$ Donor notification and post-donation counselling have a great benefit to the blood donors, to the community, and to the blood transfusion services. The main result is the awareness among donors and a decrease in the incidence of TTI in recipients. The donors are counselled first to visit the respective consultants and ICTC centres and are advised not to donate blood in the future. TTI, being an asymptomatic disease, if acquired by healthy donors can be a serious threat to the safety of the collected blood, and hence the donors must be screened for high-risk behavior prior to each donation. Disclosure of abnormal reports and the post-donation counselling process is crucial for a blood donor as it can help the donor to start the treatment and to take preventive measures for their family.

\section{CONCLUSION}

The majority of blood banks in India discard the TTI reactive units and do not inform the blood donors about the abnormal results because of the paucity of resources. In spite of the existing constraints, our centre is strictly trying to contact the sero-reactive donors as a responsibility towards them. Though the response of the contacted blood donors was less, however, we hope to achieve $100 \%$ post donation counselling. A photo identity or a donor unique identity would help a lot for notification. The majority of the responders being youngsters, it would be beneficial if we adopt more developed communication methods like social media groups, email IDs as well as strengthen our initial pre-donation counselling process, to motivate and spread awareness among blood donors with correct and verified contact details. Counselling is "a better said than done" procedure making us to learn some new things with each new donor counselled.

\section{ACKNOWLEDGEMENT}

We are immensely grateful to all the authors for data collection, analysis and preparing the manuscript.

Ethical Clearance: As we have done retrospective analysis of departmental data there is no requirement of ethical clearance.

Conflicts of interest: There are no conflicts of interest.

Financial support: Nil 


\section{REFERENCES}

1. Government of India. Drugs and Cosmetics rules. (Amended till 30th June 2005) <http://www.cdsco.nic.in/html/Drugs and Cosmetic Act.pdf $>$; (1945), accessed on 12.5.2020

2. National AIDS Control Organisation. (2007) [accessed 13.5.2020].

3. Bianco C, Kessler D. Donor notification and counselling management of blood donors with positive test results. Vox sanguinis. 1994;67:255-9.

4. National AIDS Control Organisation. National blood policy of India, 29 August (2007). <http://unpan1.unorg/intradoc/groups/ public/documents/.pdf $>$ [accessed 07.05.2020].

5. Drugs and Cosmetics Act. The Gazette of India. New Delhi: Ministry of Health and Family Welfare [accessed 2018 Mar 26].

6. Dhot, Paramjit (2003). An Action Plan for Blood Safety. National AIDS Control Organization, Ministry of Health and Family Welfare.G O I. 1-85.
7. Kleinman S, Wang B, Wu Y, Glynn SA, Williams A, Nass C, et al. Retrovirus Epidemiology Donor Study. The donor notification process from the donor's perspective. Transfusion. 2004; 44:658-66.

8. Moyer LA, Shapirog CN, Shulman G, Brugliera PD, Alter MJ. A survey of hepatitis B surface antigen positive blood donors: Degree of understanding and action taken after notification. Transfusion.1992;32:702-6.

9. G, Kaur P, Basu S, Kaur R, Sharma S. Donor notification and counselling--experience and challenges. Transfus Apher Sci.2013;49:291-4.

10. Agarwal N. Response rate of blood donors in the Uttarakhand region of India after notification of reactive test results on their blood samples. Blood Transfus.2012:1-3.

11. Choudhury LP, Tetali S. Ethical challenges in voluntary blood donation in Kerala, India. J Med Ethics. 2007;33:140-2. 